\title{
Contracepção de emergência no contexto das farmácias: revisão crítica de literatura
}

\section{| ${ }^{\text {Sabrina Pereira Paiva, }}{ }^{2}$ Elaine Reis Brandão |}

Resumen: A revisão de literatura busca discutir as políticas de provisão da contracepção de emergência (CE), notadamente por meio das farmácias, na literatura internacional e nacional. A pesquisa foi realizada nas bases de dados Medline/Pubmed, Sociological Abstracts e SciELO Brasil, considerando o período de janeiro/2005 a dezembro/2009. Os descritores utilizados foram: emergency contraception e pharmacy; incluindo-se também anticoncepção de emergência para o contexto brasileiro. Para análise, foram selecionados 36 artigos, 29 internacionais e sete nacionais. A discussão dos resultados contemplou duas perspectivas analíticas: disponibilidade e barreiras à CE nas farmácias, verificando a posição dos farmacêuticos sobre o método; e a posição das usuárias/consumidoras da $\mathrm{CE}$ a respeito do contraceptivo e seus locais de provisão, no contexto nacional e internacional. Tais estudos evidenciam que a adoção de políticas públicas favoráveis à distribuição/venda da CE pelas farmácias confere, de modo geral, avaliação positiva de farmacêuticos e usuários, principalmente devido à possibilidade de dispensar o contraceptivo de forma mais ágil. Os países que adotaram tal política discutem a necessidade de aconselhamento em saúde sexual e reprodutiva para os consumidores no âmbito da própria farmácia. Entretanto, no Brasil, o medicamento quase sempre é vendido sem receita médica e orientação do farmacêutico, pois não há política pública que inclua a provisão pelas farmácias.

> Palabras clave: Pílula do dia seguinte; anticoncepcionais pós-coito; farmácia; saúde sexual e reprodutiva.
1 Doutoranda do Instituto de Estudos em Saúde Coletiva da Universidade Federal do Rio de Janeiro. Endereço eletrônico: sabrinappaiva@gmail.com

2 Departamento de Medicina Preventiva e Instituto de Estudos em Saúde Coletiva da Universidade Federal do Rio de Janeiro. Endereço eletrônico: brandao@iesc.ufrj.br

Recebido em: 20/10/2010. Aprovado em: 25/02/2011. 
A pílula para contracepção de emergência (CE) constitui-se de compostos hormonais concentrados utilizados por curto período de tempo, atuando na suspensão da ovulação e migração do esperma, nos dias seguintes à relação sexual, tendo indicação restrita a situações especiais (BRASIL, 2006a; BRASIL, 2006b). Entre as indicações estão a relação sexual sem uso de método anticoncepcional, falha conhecida do método em uso de rotina, uso inadequado do anticonceptivo e violência sexual (FIGUEIREDO; BASTOS, 2008).

O tratamento original da $\mathrm{CE}$, contendo a combinação de estrogênio e progesterona, foi descrito, em 1972, pelo médico canadense Albert Yuzpe para prevenção da gravidez, como resposta às consequências do estupro. A partir de então, as pílulas foram tomadas de forma combinada para atingir tal objetivo, mas provocavam inúmeros efeitos adversos, como náusea, vômito e dor de cabeça. Em fins dos anos 1990, foi criado um medicamento composto por um único hormônio (progesterona), que diminuiu sensivelmente os efeitos negativos e aumentou a efetividade do método, popularmente conhecido como pílula do dia seguinte (PDS), por ser um método que atua pós-coito (RAGLAND et. al., 2009).

Estima-se que este medicamento tenha, em média, índice de efetividade de $75 \%$, podendo ser menor dependendo do regime utilizado - regime de Yuzpe, em homenagem ao médico canadense, mais antigo, adota pílulas combinadas de estrogênio e progesterona, e as pílulas de progesterona atuais -, e do tempo transcorrido do intercurso desprotegido, sendo que a cada dia diminui a sua efetividade (BRASIL, 2006a; 2006b; LEVINE; SOON, 2006; LEUNG et al., 2008). No entanto, a despeito da segurança da CE na prevenção da gravidez, permanecem inúmeras controvérsias, dificultando a obtenção do medicamento no tempo oportuno. Parte dessa problemática parece estar relacionada à posição irregular que tal medicamento ocupa no repertório de métodos disponíveis, pois é o único a ser utilizado após a relação sexual (PECHENY; TAMBURRINO, 2009).

O uso após o ato sexual levou a sua associação com medicamentos abortivos, até mesmo entre profissionais que o dispensam ou prescrevem. A mídia de massa contribui para aumentar a confusão, ao misturar preocupações sobre moralidade sexual, uso inapropriado da CE e medo do aumento das doenças sexualmente transmissíveis (DST) pela ausência do preservativo (SOUZA; BRANDÃO, 2009; BISSEL et al., 2006). Diante disso, a Organização Mundial de Saúde (OMS), 
juntamente com outras instituições atuantes no campo da saúde sexual e reprodutiva,

criou em 1995 o Consórcio Internacional de Anticoncepção de Emergência, com a intenção de desenvolver estratégias para ampliar a provisão desse método em vários países, especialmente naqueles em desenvolvimento (MARTIN, 2004).

A busca para ampliação do acesso à CE está ligada ao interesse em diminuir as taxas de gravidez na adolescência, gravidez imprevista e aborto inseguro, por isso alguns países desenvolvidos têm formulado políticas para diminuir as barreiras à CE. Dentre elas, pode-se citar a política de provisão antecipada do medicamento para as mulheres (antes do ato sexual desprotegido), e a permissão legal para que o farmacêutico forneça a CE sem receita médica, mediante cumprimento de protocolos específicos.

Desse modo, esta revisão de literatura ${ }^{1}$ tem como objetivo apresentar a discussão sobre as políticas de provisão da $\mathrm{CE}$, notadamente por meio das farmácias, expressa na produção científica internacional e nacional, como contribuição para a formulação de políticas públicas na área da assistência à contracepção no campo mais amplo dos direitos sexuais e reprodutivos.

\section{Metodologia}

Foram feitas buscas em três bases de dados do Portal de Periódicos Capes Medline/Pubmed, Sociological Abstracts e SciELO Brasil -, considerando-se o período de janeiro de 2005 a dezembro de 2009. Os descritores utilizados no levantamento de literatura foram: emergency contraception e pharmacy, incluindose anticoncepção de emergência na base nacional.

Mediante pesquisa na base de dados Medline/Pubmed, foram obtidos 96 resultados. O levantamento realizado na base Sociological Abstracts gerou oito resultados. Já para a investigação na base SciELO Brasil, utilizando-se contracepção de emergência, oito resultados; anticoncepção de emergência, 16 resultados; e com os descritores contracepção de emergência e farmácia obteve-se um resultado, mas combinando anticoncepção de emergência com farmácia não se obteve resultado.

Para seleção dos artigos, foram utilizados os seguintes critérios de inclusão: 1) resultados de pesquisas com provedores e farmacêuticos sobre a provisão da CE pelas farmácias; 2) resultados de pesquisas com usuários em relação à $\mathrm{CE}$, focando naqueles que buscam o medicamento via farmácias; 3) revisões de literatura sobre a CE. 
Para a análise, foram reunidos 34 artigos, sendo dez provenientes dos Estados Unidos, três estudos do Canadá, sete do Reino Unido, três franceses, um espanhol, dois australianos, dois latino-americanos e cinco brasileiros. Houve clara predominância de estudos populacionais em todos os países analisados, e conta-se, do total de trabalhos selecionados, com cinco investigações sob a perspectiva das ciências sociais e duas revisões sistemáticas de literatura.

Devido à escassez de estudos que contemplem o contexto das farmácias no Brasil e América Latina, foram também considerados para efeito desta revisão quatro artigos não extraídos das bases citadas, mas pertinentes ao objetivo da revisão, porque atendem devidamente a um dos três critérios de inclusão acima citados: Bergallo (2010); Figueiredo et al. (2008); Figueiredo et. al. (2007); e Pecheny e Tamburrino (2009). São dois latino-americanos e dois brasileiros, somando-se ao final 36 artigos analisados.

A apresentação e discussão dos resultados encontrados são feitas sob duas perspectivas analíticas: a) disponibilidade e barreiras à provisão da $\mathrm{CE}$ nas farmácias, verificando a posição dos farmacêuticos no contexto internacional e nacional; b) e a posição das usuárias/consumidoras da $\mathrm{CE}$ a respeito do método e seus locais de provisão no contexto nacional e internacional.

\section{Discussão dos resultados}

\section{Disponibilidade e barreiras à provisão da contracepção de emergência pelas farmácias: posição dos farmacêuticos no contexto nacional e internacional}

Quanto à disponibilidade da CE nas farmácias, há estudos do Canadá, Reino Unido, EUA e França, todos relacionados ao contexto de liberação da venda da CE sem prescrição médica pelas farmácias. Parte significativa da literatura enfatiza a discussão sobre as facilidades e dificuldades enfrentadas pelos consumidores para adquirir a CE via farmácias, considerando-se diferentes cenários (rurais, urbanos, cidades fronteiriças, etc.), conferindo a opinião dos farmacêuticos quanto à dispensa desse medicamento.

Em relação a este aspecto, os Estados Unidos são o país que apresenta maior número de estudos, alguns descritivos e de base populacional, realizados em diferentes regiōes, os quais observam que não há grande diferença em relação à disponibilidade da CE nas farmácias de municípios rurais, urbanos e de fronteira. 
Essa grande confluência de estudos, sobretudo durante a última década, refere-se ao desenvolvimento do programa Pharmacy Access em alguns estados americanos. Tal estratégia permite acesso direto à $\mathrm{CE}$ nas farmácias, com treinamento específico para farmacêuticos, que trabalham de acordo com protocolos de acordo mútuo (BIGBEE et al., 2007). Esta legislação é original de 1979, e permite ao farmacêutico desenvolver acordos colaborativos com a categoria médica, possibilitando ao primeiro o direito de prescrever certas drogas descritas no acordo, passando por revisão a cada dois anos (SOON et al., 2004).

Em agosto de 2006, o Food and Drug Administration permitiu aos farmacêuticos vender a CE sem prescrição médica para mulheres com idade superior ou igual a 18 anos. Essa decisão aumentou a probabilidade de a mulher obtê-la em tempo oportuno. Entretanto, nem sempre elas conseguem adquiri-la por essa via, já que os farmacêuticos podem optar por não vender o medicamento, baseando-se na cláusula da objeção consciente, segundo a qual o farmacêutico está autorizado a negar a dispensa de qualquer medicamento, caso este colida com sua ética pessoal (SHACTER et al., 2007).

Observa-se nesse país a baixa disponibilidade desse medicamento nas farmácias e as razões mais alegadas pelos farmacêuticos são: falta de demanda, de treinamento dos farmacêuticos para aconselhamento em reprodução e preocupações quanto ao impacto negativo do medicamento no organismo. Embora não tenham sido a tônica principal do discurso, houve relatos relacionados às objeçôes morais/religiosas em dispensar o medicamento, principalmente para o público jovem (SAMPSON et al., 2009; BIGBEE et al., 2007; FRENCH; KAUNITZ, 2007; SHACTER et al., 2007; CHUANG; SHANK, 2006; RIPER; HELLERSTEDT, 2005).

Como observam Chuang e Shank (2006), embora não tenham sido encontradas diferenças significativas entre farmácias urbanas, rurais e de fronteira, as mulheres rurais enfrentam barreiras relacionadas ao tempo em que as farmácias ficam abertas, sobretudo nos fins de semana, e quanto à impossibilidade de anonimato. Essa característica dos estabelecimentos é normalmente considerada relevante para as usuárias da CE, sobretudo as jovens, que estão mais vulneráveis à gravidez imprevista e ao aborto.

O estudo de Shacter et al. (2007) teve como objetivo determinar a disponibilidade da CE nas farmácias das três maiores áreas metropolitanas dos 
Estados Unidos, incluindo-se estados com diferentes políticas relacionadas à recusa dos farmacêuticos em dispensar o contraceptivo. A hipótese dos autores, de que a política de estado que permite aos farmacêuticos se apoiar na cláusula da objeção consciente está associada à baixa disponibilidade da CE nas farmácias, foi confirmada. Demonstra-se a forte ligação existente entre política do estado e disponibilidade da CE na farmácia.

A investigação realizada por Sampson et al. (2009) contemplou entrevistas em profundidade, revelando aspectos relativos à motivação do farmacêutico para dispensar a CE. Enquanto alguns farmacêuticos têm vagas preocupações quanto ao impacto negativo do uso do método nas adolescentes, a preocupação mais frequente se relaciona à necessidade de mais tempo para aconselhamento do público a respeito do método. Percebe-se nesse país a melhoria da disponibilidade da CE após a desregulação e devido a programas como o Pharmacy Access. Entretanto, há determinado número de farmácias que não dispõe de estoque de CE. A possibilidade aberta pela cláusula da objeção consciente levantou um debate bastante acirrado sobre a ética profissional versus ética pessoal/subjetiva.

No Canadá, dois estudos selecionados referem-se a avaliações sobre os efeitos da política de disponibilização da CE nas farmácias sem prescrição médica, a partir de 2000. Ambos são de base populacional e demonstram que a provisão do método via farmácias ampliou o acesso, principalmente em áreas rurais, por conta do tempo maior de atendimento das farmácias e do maior número delas em relação aos serviços de planejamento familiar ou outros serviços (SOON et al., 2005; DUNN et al., 2008).

No Reino Unido, a iniciativa de tornar disponível a CE pelas farmácias faz parte de um projeto nacional sobre Saúde Sexual e HIV, cujo objetivo é reduzir em torno de 50\% a gravidez em adolescentes, até o ano de 2010 (LLOYD; GALE, 2005).

O apelo para a disponibilidade da CE nas farmácias cresceu principalmente a partir de 1999, com o projeto Patient Group Direction (PGD), que facilitou o acesso livre ao medicamento. O protocolo PGD provê autoridade legal para que o farmacêutico dispense o contraceptivo. Nesse caso, o medicamento é adquirido sem custos para o usuário. A partir de 2001, criou-se um segundo mecanismo para ampliar a disponibilidade deste contraceptivo através das farmácias, a venda livre, ou seja, o medicamento fica disponível para venda sem que seja necessária 
a solicitação e intervenção do farmacêutico - over-the-counter. Esta estratégia permite que o medicamento seja adquirido por mulheres com idade igual ou superior a 16 anos e, nesse caso, o consumidor precisa pagar pelo contraceptivo (BLACK et al., 2008).

O estudo de Loyd e Gale (2005), com enfoque populacional, verificou aumento da provisão da $\mathrm{CE}$ por meio das farmácias em duas regiōes rurais do Reino Unido. A principal conclusão é que a provisão pelas farmácias aumentou a possibilidade de escolha do usuário e o acesso ao medicamento, o que resultou em crescimento global do fornecimento da CE nessas áreas.

A investigação de Bissell et al. (2006) analisa, a partir da perspectiva dos farmacêuticos, a viabilização da CE pelas farmácias, segundo o protocolo PGD. De modo geral, os farmacêuticos entrevistados destacam os benefícios para as mulheres, relacionando-os a: preocupação em evitar que seus médicos generalistas saibam que necessitaram utilizar a CE; ampliação do acesso ao medicamento pela diminuição do tempo necessário para obtê-lo; ausência de custos para mulheres que adquirem o medicamento via PGD. Entretanto, os farmacêuticos também expressam certas reservas, pelo receio de ocorrer aumento do uso repetido do método e de DST e dos possíveis impactos negativos sobre o comportamento contraceptivo de rotina (BISSELL et al., 2006).

O estudo de Cooper et al. (2008) foca sua preocupação nas questóes práticas, religiosas e éticas relacionadas à dispensa da $\mathrm{CE}$ pelos farmacêuticos. Foram identificados três tipos de farmacêuticos: os que não têm problemas éticos em dispensar a CE; aqueles completamente opostos a seu provimento; e os que decidiram contingencialmente dispensar o medicamento somente em algumas situações. Uma preocupação dessa pesquisa se refere à confusão generalizada a respeito da terminologia apropriada e ação farmacológica da CE. Muitos profissionais continuam usando o termo pílula do dia seguinte, que pode causar equívocos. Novamente, surge a ideia de que a provisão via farmácias poderia levar ao uso frequente e ao aumento de DST (COOPER et al., 2008).

Pesquisa realizada por Delotte et al. (2008) na região de Nice, na França, avaliou a aplicação do Decreto 2.020-39 (o qual obriga dispensa da CE nas farmácias sem receita médica e custos para menores de 18 anos), por meio da seleção aleatória de 53 farmácias. Pouco mais da metade das farmácias entregou o método gratuitamente. Além disso, na maior parte dos estabelecimentos 
visitados, não houve possibilidade de conversa reservada com as jovens, indicando desconforto maior para essa faixa etária adquirir o medicamento.

A pesquisa de Moreau et al. (2006), em contexto francês, evidencia que a introdução da $\mathrm{CE}$ como produto disponível, sem prescrição, tem resultado em significativo crescimento de seu uso nos últimos cinco anos, especialmente entre mulheres abaixo de 25 anos. Em 2004, a maioria das mulheres (85\%) obteve a CE diretamente nas farmácias.

Através desses estudos, percebe-se que o Canadá, Reino Unido e França apresentam perspectiva similar, no que se refere à ampliação do acesso à CE a partir da possibilidade de venda sem prescrição, especificamente para as mulheres que moram em áreas rurais. A provisão via farmácias também produziu impactos positivos para as populaçōes urbanas, que podem utilizá-las no período noturno. Nesses países, incluindo-se alguns estados norte-americanos, considera-se que os farmacêuticos têm potencial para contribuir para a melhoria da saúde pública, cumprindo papel fundamental na provisão do medicamento, pois dispensam com mais agilidade, questão crucial no caso da CE (SOON et al., 2004).

A América Latina tem um cenário bastante diversificado. Grupos políticos e afiliados à Igreja Católica representam grande força oponente à CE na esfera político-ideológica. Devido às objeções desses setores, comumente ocorrem dificuldades no cumprimento da legislação e distribuição desse medicamento nos serviços públicos de saúde e farmácias (SOUZA; BRANDÃO, 2009; BERGALLO, 2010; FAÚNDES et. al., 2007).

No Brasil, com relação à provisão da CE pelas farmácias, aprovou-se em 1996, por meio da Agência Nacional de Vigilância Sanitária (ANVISA), a comercialização da CE com prescrição médica. Há atualmente mais de dez marcas disponíveis no mercado, e é possível perceber o ressurgimento do debate em torno desse medicamento, por conta da facilidade de sua aquisição comercial, sem a necessidade da receita (BASTOS et al., 2009).

Contudo, a estratégia política utilizada nos países europeus e América do Norte - que inclui a farmácia como local privilegiado para o acesso à CE e permite sua dispensação sem receita médica e com aconselhamento sexual e reprodutivo do farmacêutico - encontra-se distante do que temos hoje no Brasil. Aqui houve apenas um pronunciamento do Ministro da Saúde, no jornal Folha de S. Paulo (2007), favorável à suspensão da prescrição médica para provisão da 
$\mathrm{CE}$, mas que não obteve repercussão em termos de ampliar o debate sobre outras

formas de acesso à CE. No entanto, se a maior parte das consumidoras busca tal via, é urgente pensar na diversificação das políticas de acesso ao método.

Estudo realizado por Bastos e colaboradores (2009), no contexto de um projeto de intervenção educativa voltado à prevenção de DST/Aids, com profissionais de farmácias e drogarias da área metropolitana de São Paulo, demonstrou o desconhecimento destes sobre a CE. Os autores assinalam a necessidade de incorporação de estratégias educativas no contexto das farmácias, focando na prevenção de gravidez e DST/Aids, por meio da proposição de condutas eficazes de saúde para a população.

Por fim, vale ressaltar que a literatura internacional não corrobora a afirmação de que a disponibilidade levaria ao aumento do uso repetido do medicamento, e também parece não afetar o uso do contraceptivo de rotina nem causar aumento de DST. Entretanto, há evidências crescentes de que a provisão da CE via farmácias não contribuiu até agora para diminuir as taxas de aborto em alguns países, o que talvez signifique que a extensão da venda da CE nas farmácias não atingiu o público-alvo da política, ou seja, mulheres jovens em maior risco de gravidez imprevista (BISSELL et al., 2006; RAINE et al., 2005; ROS et al., 2009).

\section{Posição das usuárias/consumidoras da $\mathrm{CE}$ a respeito do método e seus locais de provisão no contexto nacional e internacional}

Nos Estados Unidos, um estudo populacional investigou mulheres que buscam a CE em farmácias. Os resultados mostram que o dia mais comum para compra da pílula é domingo, e quase metade relatou sexo desprotegido entre sextafeira e domingo. Salienta-se a importância de se pensar que o uso da CE pode estar mais relacionado às relações esporádicas, não planejadas, vivenciadas principalmente pelos jovens (FOSTER et al., 2006). O estudo conclui ainda, em consonância com inúmeras pesquisas nacionais e internacionais, que a falta de conhecimento sobre $\mathrm{CE}$, seu modo de funcionamento no organismo e locais onde se pode adquiri-la se mantêm como barreiras de acesso ao medicamento dentro do prazo de 120 horas. A respeito do conhecimento, ocorre confusão desse método com medicamento abortivo (FOSTER et al., 2006; BALDWIN, 2008; ROCCA, 2007; SHOVELLER et al. 2007; GOULARD et al., 2006; MOHORIC-STATE; COSTA, 2009; PECHENY; TAMBURRINO, 2009; 
FIGUEIREDO et al.(org.), 2008; COSTA et al., 2008; SOUZA; BRANDÃO, 2009; ARAÚJO et al., 2009, ROS et al., 2009).

Além desse equívoco, observações moralizantes - baseadas em pareceres negativos sobre o comportamento sexual e saúde reprodutiva das mulheres que recorrem ao uso da $\mathrm{CE}$ - são verificadas em algumas pesquisas. Tais observaçōes constituem barreira potencial ao uso do contraceptivo (SHOVELLER et al., 2007; WILLIAMSON et al., 2009; MOHORIC-STATE; COSTA, 2009; PECHENY; TAMBURRINO, 2009). Assim, as pesquisas citadas revelam que os momentos de vulnerabilidade, em que se necessita da CE, podem ser avaliados diferentemente pelas potenciais usuárias da CE. As mulheres relatam maior culpabilidade quando há esquecimento e não-uso do preservativo. Algumas mulheres confessam preferir ocultar do profissional quando deixaram de utilizálo. Ao contrário, caso ocorra o rompimento do preservativo, elas não se culpam ou se envergonham (SHOVELLER et al., 2007; WILLIAMSON et al., 2009; PECHENY; TAMBURRINO, 2009).

Salienta-se que o grau de vergonha e culpa, principalmente das jovens, é ampliado quando o provedor médico ou farmacêutico as conhece. A questão é mais crítica para pessoas que vivem em regiōes rurais, onde muitas vezes só há um serviço de saúde disponível e uma farmácia, sendo os provedores conhecidos das pessoas que necessitam da CE (MOHORIC-STATE; COSTA, 2009; PECHENY; TAMBURRINO, 2009).

Ressalta-se pesquisa recente (WILLIAMSON et al., 2009) realizada na Escócia, que explora as percepções de mulheres jovens sobre o uso de CE e suas experiências com sexo desprotegido, discutindo as razões pelas quais o acesso à CE pode não ter sido suficiente para reduzir as taxas de gravidez imprevista e aborto nessa região. $\mathrm{O}$ estudo evidencia que é mais comum o uso da CE após falha no contraceptivo do que após experiência de sexo desprotegido, podendo significar bias de conveniência social nas respostas das mulheres. Embora não se possam generalizar os resultados dessa pesquisa, ela lança luzes para futuras investigações que busquem explicar por que o crescimento da provisão da $\mathrm{CE}$ em diferentes locais de acesso tem falhado em reduzir as taxas de aborto e gravidez em alguns países.

A pesquisa realizada por Ros et al. (2009) na Espanha traz observaçôes que ajudam a compreender a questão colocada acima, pois identifica que o perfil das mulheres que usam a CE naquele país é particularmente diferente das mulheres 
que realizam o aborto, as quais, em geral, têm menor nível socioeconômico e estão mais expostas à gravidez imprevista.

Pode-se citar ainda, em relação a esse aspecto, pesquisa realizada por Keogh (2005) na Austrália, cuja intenção foi compreender, mediante entrevistas em profundidade, a situação que levou ao uso da $\mathrm{CE}$, a decisão e experiência de usálo e as consequências para o uso dos contraceptivos de rotina. Tornou-se claro, a partir das entrevistas, que o uso da CE envolve mais do que simples falha no contraceptivo, pois implica uma série de decisões inter-relacionadas e que têm diferentes significados. A pesquisa define tipos de usuárias da $\mathrm{CE}$, englobando desde as que têm alto controle sobre sua saúde sexual e reprodutiva até as que têm controle dificultado por inúmeras razões (KEOGH, 2005).

Sobre as preferências de locais de acesso à CE, os estudos evidenciam comparação entre o cenário das clínicas de planejamento familiar, clínicas gerais e farmácias. Alguns países, por desenvolverem a política de provisão antecipada da $\mathrm{CE}$, também comparam padrões de uso, diferenciando o acesso à $\mathrm{CE}$ após e antes da relação sexual (ROCCA et al., 2007; SHOVELLER et al., 2007; LEWINGTON; MARSHALL, 2006; SESTON et al., 2007; BLACK et al., 2008; MOREAU et al., 2006; GOULARD et al., 2006).

De modo geral, as farmácias representam alternativa fundamental para as usuárias, já que podem permanecer abertas por mais tempo, facilitando a obtenção do medicamento, comparando com outros locais de acesso. Contudo, em relação às preferências, predominam as clínicas de planejamento familiar, dada a possibilidade de privacidade, de conversa mais extensa com o profissional sobre contracepção e de obter informações sobre a CE (LEWINGTON; MARSHALL, 2006; BLACK et al., 2008; ROCCA et al., 2007).

O estudo realizado por Seston et al. (2007), na Inglaterra, objetivou identificar quais atributos dos serviços as mulheres consideram importantes: tempo diário de funcionamento; consulta médica; custo, tempo de espera; privacidade na consulta; atitudes do profissional (SESTON et al., 2007). Os resultados demonstram que os principais fatores considerados para escolha das mulheres pelos serviços são as atitudes dos profissionais, privacidade na consulta e orientação recebida de um médico ou enfermeira, ficando o farmacêutico em posição secundária. Outro dado interessante se refere ao fato de que, se as mulheres não se sentem satisfeitas com alguns aspectos do serviço procurado, elas preferem não utilizá-lo, mesmo correndo 
risco de engravidar. Além disso, a manutenção da privacidade é importante para elas, sobretudo as mais jovens. Os custos são identificados como barreiras para adquirirem a CE via farmácias (SESTON et al., 2007).

No Canadá, o estudo desenvolvido por Shoveller et al. (2007) investigou as barreiras quanto ao uso da CE entre mulheres jovens de diferentes contextos socioculturais na Colúmbia Britânica. Realizaram-se entrevistas em profundidade e, de todas as entrevistadas, somente duas obtiveram a CE via farmácia. Entre as participantes, somente cinco mulheres atestaram conhecer as mudanças na autoridade prescritiva da CE.

Quanto à realidade latino-americana, as diferenças com os países desenvolvidos se destacam pelo simples fato de não haver muitas alternativas de provisão do método, além da farmácia para o consumidor (FIGUEIREDO et. al., 2008b; SOUZA; BRANDÃO, 2009). Neste contexto, a discussão sobre o acesso à CE é mais restrita, não havendo uma política pública voltada à inclusão das farmácias como local de acesso à $\mathrm{CE}$, com responsabilidade social e cumprimento de protocolos específicos entre farmacêuticos e médicos, visando a garantir o atendimento orientado em saúde sexual e reprodutiva (FAÚNDES et al., 2007; FIGUEIREDO et al., 2007; PECHENY; TAMBURRINO, 2009).

Nos serviços públicos de saúde, os valores morais e religiosos dos provedores e formuladores de políticas, o julgamento moral de profissionais de saúde, acabam por obstaculizar às vezes o acesso à CE pela população em situação de desigualdade econômica, a qual é mais vulnerável, devido à maior fertilidade e maior frequência de gravidez imprevista (SOUZA; BRANDÃO, 2009). O fato é que o uso da CE cresceu consideravelmente no Brasil, entre 1996 e 2006, segundo a Pesquisa Nacional de Demografia e Saúde da Criança e da Mulher (PNDS). O uso da CE não apareceu na PNDS de 1996, mas, em 2006, 12\% das mulheres de 15 a 49 anos afirmaram ter utilizado esse método, cuja adesão teve maior representatividade na faixa etária de 20 a 24 anos (18,5\%), e 10,4\% entre mulheres de 15 a 19 anos. A CE passou a ocupar, em 2006, o quinto lugar entre todos os métodos contraceptivos usados e o terceiro entre mulheres não unidas e ativas sexualmente (BRASIL/MS, 2008).

No Brasil, há urgência de se discutir essa temática, pois tal medicamento é vendido cotidianamente, sem monitoramento apropriado das autoridades sanitárias para que se possa acompanhar os desdobramentos na saúde sexual 
e reprodutiva de seus consumidores. Não se sabe se as consumidoras estão

devidamente orientadas quanto ao uso da CE, se sofrem efeitos colaterais, se utilizam outros métodos concomitantes etc.

\section{Considerações finais}

Na literatura considerada, os países que adotaram uma política pública de acesso à contracepção de emergência pelas farmácias, seja através dos protocolos de acordo mútuo entre farmacêuticos e médicos ou pela liberação da venda sem prescrição, recebem, de modo geral, avaliação positiva de farmacêuticos e usuários. Tal avaliação se refere sobretudo à possibilidade de adquirir o contraceptivo de forma mais ágil do que se a usuária tivesse que conseguir a receita médica ou adquiri-lo diretamente nos serviços públicos de saúde.

Além disso, em todos os países em que tal política foi adotada houve preocupação com a necessidade de os consumidores receberem aconselhamento em saúde sexual e reprodutiva. Adotar a dispensação pelas farmácias como uma medida de melhoria do acesso ao medicamento não significou perder de vista as implicações do uso inapropriado da CE, como o próprio nome indica, devendo ser utilizada apenas em situações emergenciais. Portanto, faz-se necessária a ampliação do debate sobre a garantia de acesso e uso racional da $\mathrm{CE}$, incluindo os farmacêuticos nessa discussão, pois eles são aliados importantes neste tema estratégico à saúde pública.

O desconhecimento dos usuários sobre o modo de atuação da CE no organismo feminino e a confusão com outros medicamentos de efeito abortivo foram aspectos recorrentes nos trabalhos analisados. Qualquer proposta de educação sexual voltada aos adolescentes e jovens não pode prescindir de debater a CE (FOSTER et al., 2006; BALDWIN et al., 2008; ROCCA et al., 2007; SHOVELLER et al. 2007; GOULARD et al., 2006; MOHORIC-STATE; COSTA, 2009; PECHENY; TAMBURRINO, 2009; FIGUEIREDO et al., 2008; COSTA et al., 2008; SOUZA; BRANDÁO, 2009; ARAÚJO et al., 2009; BASTOS et. al., 2009).

Conforme alguns estudos apontam, a CE é um método que as mulheres consideram que deve ser mantido em segredo, pois elas sentem embaraço, vergonha e culpa por fazerem uso dele. Nem mesmo a possibilidade de adquirir o método diretamente nas farmácias contribuiu para que isso mudasse completamente (SHOVELLER et al., 2007; KEOGH, 2005). Nesse sentido, percebe-se que o grau 
de constrangimento sentido pelas mulheres pode estar relacionado aos momentos de vulnerabilidade por elas vividos em relação ao (des)controle da contracepção. Tal vulnerabilidade está ligada à manutenção da hierarquia de gênero (dificuldades de negociação com o parceiro a respeito do método contraceptivo) e por injunções do relacionamento afetivo. Portanto, mesmo que se tenha um grau ótimo de informação, que se mantenha uma vida sexual protegida de possíveis "riscos", podem ocorrer momentos em que o uso da CE torna-se imprescindível. ${ }^{2}$

\section{Referências}

ANDERSON, C.; BLENKINSOPP, A. Community pharmacy supply of emergency hormonal contraception: a structured literature review of international evidence. Human Reproduction, v.21, n.1, p.272-284, 2006.

ARAÚJO, M.S.P. et al. Comportamento sexual e contracepção de emergência entre adolescentes de escolas públicas de Pernambuco, Brasil. Cadernos de Saúde Pública, v.25, n.3, p.551-562, 2009.

BALDWIN, S. et al. Who is using emergency contraception? Awareness and use of emergency contraception among California women and teens. Women's Health Issues, v.18, p.360-368, 2008.

BASTOS, M.R. et al. Práticas contraceptivas entre jovens universitárias: o uso da anticoncepção de emergência. Texto e Contexto Enfermagem, Florianópolis, v.17, n.3, p.447-56, 2008.

BASTOS, S. et al. Prevenção de doenças sexualmente transmissíveis e procura da contracepção de emergência em farmácias e drogarias do município de São Paulo. Saúde sociedade, São Paulo, v.18, n.4, p.787-799, 2009.

BERGALLO, P. El debate jurídico en torno a la anticoncepción de emergencia: una Mirada comparada. In: ARILHA, M. et al. (orgs). Contracepção de emergência no Brasil e América Latina: dinâmicas políticas e direitos sexuais e reprodutivos. São Paulo: Oficina Editorial, 2010. p. 09-62.

BIGBEE, J. et al. Pharmacy access to emergency contraception in rural and frontier communities. National Rural Health Association, v.23, n.4, p.294-298, 2007.

BISSEL, P.; SAVAGE, I.; ANDERSON, C. A qualitative study of pharmacists perspectives on the supply of emergency hormonal contraception via patient group direction in the UK. Contraception, v.73, p.265-270, 2006.

BLACK, K. et al. Provision of emergency contraception: a pilot study comparing access through pharmacies and clinical settings. Contraception, v.77, p.181-185, 2008.

BRASIL. Ministério da Saúde. Pesquisa nacional de demografia e saúde da criança e da mulher-PNDS, 2006. Relatório. Brasília: MS, 2008. 583p. 
BRASIL. Ministério da Saúde. Anticoncepção de emergência: perguntas e respostas para profissionais de saúde. Brasília: MS, 2006a.

BRASIL. Ministério da Saúde. Cartilha de Direitos Sexuais e Reprodutivos: uma prioridade de governo. Brasília: MS, 25p, 2005.

BRASIL. Ministério da Saúde. Nota técnica sobre anticoncepção de emergência. Brasília: MS, 2006b.

CHUANG, C.; SHANK, L. Availability of emergency contraception at rural and urban pharmacies in Pennsylvania. Contraception, v.73, p.382-385, 2006.

COOPER, R.; BISSELL, P.; WINGFIELD, J. Ethical, religious and factual beliefs about the supply of emergency hormonal contraception by UK community pharmacists. J Fam Plann Reprod Health Care, v.34, n.1, p.47-50, 2008.

COSTA, N.F.P. et al. Acesso à anticoncepção de emergência: velhas barreiras e novas questôes. Rev. Bras. Ginecologia e Obstetrícia, Rio de Janeiro, v.30, n.2, p.55-60, 2008.

DELOTTE, J. et al. Délivrance de la contraception d'urgence aux mineures dans les pharmacies françaises. Gynécologie, obstétrique \& Fertilité, v.36, p.63-66, 2008.

DÍAZ, S. et al. Acceptability of emergency contraception in Brazil, Chile, and Mexico. 1Perceptions of emergency oral contraceptives. Cad. Saúde Pública, Rio de Janeiro, v.19, n.5, p.1507-1517, 2003.

DUNN, S.; BROWN, T.; ALLDRED, J. Availability of emergency contraception after its deregulation from prescription-only status: a survey of Ontario pharmacies. CMAJ, v.178, n.4, p.423-424, 2008.

FAÚNDES, A. et al. Emergency contraception under attack in Latin America: response of the medical establishment and civil society. American Reproductive Health Matters, v.15, n.29, p.130-138, 2007.

FIGUEIREDO, R.; BASTOS, S. Contracepção de emergência: atualização, abordagem, adoção e impactos em estratégias de DST/AIDS. São Paulo: Instituto de Saúde, 2008. 52 p. FIGUEIREDO, R., et al. (Org.). Comportamento sexual, uso de preservativos e contracepção de emergência por adolescentes do município de São Paulo: estudo com estudantes de escolas públicas de ensino médio. São Paulo, Instituto de Saúde, 2008. 38p.

FIGUEIREDO, R. et al. Distribuição da contracepção de emergência na atenção básica de São Paulo: caracterização de oferta em PSF e UBS dos municípios do estado. Bol. Instit. Saúde, São Paulo, n.42, 2007.

FOLHA de S. Paulo. "Governo quer facilitar uso da pílula do dia seguinte”. 26 jun 2007.

FOSTER, D. et al. Pharmacy access to emergency contraception in California. Perspectives on Sexual Reprod. Health, v.38, n.1, p.46-52, 2006.

FRENCH, K.; KAUNITZ, M. Pharmacy access to emergency contraception in Jacksonville, FL: a secret shopper survey. Contraception, v.75, p.126-130, 2007. 
GOULARD, H. et al. Contraceptive failures and determinants of emergency contraception use. Contraception, v.74, p.208-213, 2006.

KEOGH, L. A qualitative study of women's use of emergency contraception. J Fam Plann Reprod Health Care, v.31, n.4, p.288-293, 2005.

LEUNG, V.W.Y.; SOON, J.A.; LEVINE, M. Emergency contraception update: a Canadian perspective. Clinical pharmacology \& therapeutic, v. 83, n.1, p.177-180, 2008.

LEVINE, M.; SOON, J.A. Risk of pregnancy among women seeking emergency contraceptives from pharmacists in British Columbia. J Obstet gynaecol Can, v.28, n.1, p. 879-883, 2006.

LEWINGTON, G.; MARSHALL, K. Access to emergency hormonal contraception from community pharmacies and family planning clinics. British Journal of Clinical Pharmacology, v.61, n.5, p.605-608, 2006.

LLOYD, K.; GALE, E. Provision of emergency hormonal contraception through community pharmacies in rural area. J Fam Plann Reprod Health Care, v.31, n.4, p.297-300, 2005.

MARTIN, A. La anticoncepción de emergencia en América Latina y el Caribe. Rev. Panamericana de Salud Pública, v.16, n.6, p.424-431, 2004.

MOHORIC-STATE, D.; DE COSTA, C. Knowledge of emergency contraception amongst tertiary students in far North Queensland. Australian and New Zealand Journal of Obstetrics and Gynaecology, v.49, p.307-311, 2009.

MOREAU, C.; TRUSSELL, J.; BAJOS, N. The determinants and circumstances of use of emergency contraceptive pills in France in the context of direct pharmacy access. Contraception, v.74, p.476-482, 2006.

PECHENY, M.; TAMBURRINO, M.C. ¿"La palabra lo dice”? Interpretaciones cruzadas y obstáculos al acceso a a anticoncepción de emergencia. Sexualidad, Salud y SociedadRevista Latinoamericana, v.1, p.158-76, 2009.

POLIS, C. et al. Advance provision of emergency contraception for pregnancy prevention: a meta-Analysis. Obstetrics \& Gynecology. V.110, n.6, p.1379-1388, 2007.

RAGLAND, D.; WEST, D. Pharmacy students' knowledge, attitudes, and behaviors regarding emergency contraception. American Journal Pharm Educ, v.73, n.2, p.1-4, 2009.

RAINE, T. et al. Direct access to emergency contraception through pharmacies and effect on unintended pregnancy and STIs: a randomized controlled trial. Jama, v.293, n.1, p.54-62, 2005.

RIPER, K.; HELLERSTEDT, W. Emergency contraceptive pills: dispensing practices, knowledge and attitudes of South Dakota Pharmacists. Perspectives on Sexual and Reproductive Health, v.37, n.1, p.19-24, 2005. 
ROCCA, C. et al. Beyond access: acceptability, use and nonuse of emergency contraception among young women. American Journal of Obstetrics \& Gynecology, v.196, p.29, 2007.

ROS, C.; MIRET M.; RUE, M. Estudio descriptivo sobre el uso de la anticoncepción de emergencia en Catalunã. Comparación entre una zona rural y una urbana. GacSanit., v.23, n.6, p.496-500, 2009.

SAMPSON, O. et al. Barriers to adolescents' getting emergency contraception through Pharmacy access in California: differences by language and region. Perspectives on Sexual and Reproductive Health, v.41, n.2, p.110-118, 2009.

SESTON, E. et al. Women's preferences for the provision of emergency hormonal contraception services. Pharm World Sci, v.29, p.183-189, 2007.

SHACTER, H., GEE, R., LONG, J. Variation in availability of emergency contraception in pharmacies. Contraception, v.75, p.214-217, 2007.

SHOVELLER, J. et al. Identifying barriers to emergency contraception use among young women from various sociocultural groups in British Columbia, Canada. Perspectives on Sexual and Reprod Health, v.39, n.1, p.13-20, 2007.

SOON, J. et al. Effects of making emergency contraception available without a physician's prescription: a population-based study. JAMC, v.172, n.7, p.878-883, 2005.

SOON, J.A. et al. Provision of emergency contraceptives by pharmacists. British Columbia experience a template for other provinces. CPJ/RPC. v.137, n. 6, p.23-29, 2004.

SOUZA, R.A.; BRANDÃO, E. R. Marcos normativos da anticoncepção de emergência e as dificuldades de sua institucionalização nos serviços públicos de saúde. Physis, v.19, n.4, 2009.

WHITTAKER, P.; ARMSTRONG, K.; ADAMS, J. Implementing advance emergency contraception policy: what happens in the real world? Perspectives on Sexual and Reproductive Health, v.40, n.3, p.162-170, 2008.

WILLIAMSON, L.; BUSTON, K.; SWEETING, H. Young women's perceptions of pregnancy risk and use of emergency contraception: findings from a qualitative study. Contraception, v.79, p.310-315, 2009.

\section{Notas}

${ }^{1}$ A pesquisa Contracepção de Emergência: o desafio para torná-la um dispositivo institucionalizado na rede pública de serviços de saúde no Brasil conta com apoio do Programa "Jovem Cientista do Nosso Estado", Edital Faperj nº.28/2008.

${ }^{2}$ S.P. Paiva foi responsável pela elaboração, coleta, análise e interpretação dos dados e redação do artigo; E.R. Brandão foi responsável pela elaboração do estudo, análise e interpretação dos dados e revisão do texto. 


\section{Emergency contraception in the context of pharmacies: critical literature review}

This literature review aims to discuss policies for provision of emergency contraception (EC), especially through pharmacies, in the international and national literature. Research was conducted in Medline/Pubmed, Sociological Abstracts and SciELO Brazil, considering the period of the January/2005-December/2009, using the terms: emergency contraception and pharmacy, and also emergency contraception for the Brazilian context. For analysis, we selected 36 articles, 29 international and seven national. The discussion of the results included two analytical perspectives: availability and barriers to EC in pharmacies, focusing on the position of pharmacists on the method, and the position of users of emergency contraception and the supplies sites, in the national and international context. Studies show that the adoption of policies favorable to the distribution of emergency contraception in pharmacies with a prescription for pharmacists generally provides positive assessment of pharmacists and users, mainly by dispensing more agile method. The adoption of this policy has generated the need for counseling on sexual and reproductive health consumers in the pharmacy. However, this strategy has not been suggested in Brazil, where the drug is often sold without a prescription and guidance of the pharmacist as there is no specific policy in this regard.

> Key words: Emergency contraceptive; postcoital contraceptives; pharmacy; sexual and reproductive health 